\title{
Genesi e forma di un libro di poesia: L'amaro miele
}

Genèse et forme d'un recueil de poèmes: L'amaro miele

Genesis and Form of a Collection of Poems: L'amaro miele

\section{Antonio Di Silvestro}

\section{OpenEdition}

\section{Journals}

Edizione digitale

URL: http://journals.openedition.org/cei/7036

DOI: 10.4000/cei.7036

ISSN: 2260-779X

\section{Editore}

UGA Éditions/Université Grenoble Alpes

\section{Edizione cartacea}

ISBN: 978-2-37747-173-7

ISSN: $1770-9571$

Notizia bibliografica digitale

Antonio Di Silvestro, « Genesi e forma di un libro di poesia: L'amaro miele », Cahiers d'études italiennes

[Online], 30 | 2020, Messo online il 01 mars 2020, consultato il 06 mars 2020. URL : http:// journals.openedition.org/cei/7036 ; DOI : https://doi.org/10.4000/cei.7036

Questo documento è stato generato automaticamente il 6 marzo 2020

(c) ELLUG 


\title{
Genesi e forma di un libro di poesia: L'amaro miele
}

\author{
Genèse et forme d'un recueil de poèmes : L'amaro miele \\ Genesis and Form of a Collection of Poems: L'amaro miele
}

Antonio Di Silvestro

\section{Premessa}

1 Quella di apparire sulla scena letteraria come poeta 'tardivo' è una scelta che Bufalino persegue per ragioni assai distanti, ma nel profondo convergenti: per un verso l'abbandono di quella «Vita nova turgida e mortuaria» che avrebbe dovuto essere il suo primo romanzo, concepito peraltro in quegli stessi anni della "glaciazione neorealista» ${ }^{1}$ a cui risalgono le prime poesie su rivista; per un altro, la fedeltà a un impulso lirico della scrittura che, accantonata l'idea di un "poemetto narrativo» ${ }^{2}$ (venutosi a complicare di istanze da Bildungsroman e delle componenti funebri di un barocco isolano), recupera gli intermezzi poetici di Diceria dell'untore, tentando di innestarli su una serie di testi concepiti nell'immediato dopoguerra e fino a metà degli anni ' 50 (1944-1954).

2 Basterebbe questo ad avvalorare la scaturigine poetica, la tensione lirico-fantastica della scrittura e dell'opera del comisano, a cui sembra contraddire la difficoltà di creare una compagine che potesse assumere la veste di un moderno libro di poesia ${ }^{3}$, tale da evitare qualsiasi sbrigativo approdo a costruzioni raccogliticce, per addizioni irrelate di reperti poetici disseminati nel tempo.

3 Ciò che segna il corpo di parole di un libro come L'amaro miele è il suo voluto anacronismo, la ricercata sfasatura di un messaggio che suona come (volutamente) inattuale, ponendo il lettore di un Bufalino da poco esordiente di fronte a testi frutto di una «lunga attesa e persuasione di morte all'ombra grave della guerra» ${ }^{4}$, ma anche del ristoro procurato dal rassicurante ritorno e dal confortante abbraccio dell'isola-madre. 
4 In un'intervista della fine degli anni '80 l'autore non lesinava dimostrazioni di affetto per quella che era stata «la sua unica opera per decenni», professando un'immutata fedeltà a essa ${ }^{5}$. Ma quando nasce questo libro? 0 meglio, il nucleo ispirativo era già ai suoi primordi volto a costruire una sequenza lirica che contemperasse in sé temi e ragioni di un libro? Sulle date Bufalino non si smentisce: infatti, in una lettera di risposta a Luciano Anceschi, al quale aveva inviato nel marzo del'63 un fascicolo dattiloscritto delle sue poesie per riceverne un parere, egli parla di versi «vecchi di dieci anni» ${ }^{6}$, risalenti dunque agli anni 1944-1954 indicati nella Nota alla prima edizione. Se già in questa sede, mitizzando quasi petrarchescamente il salvataggio delle sue nugae giovanili dalla periodica combustione delle carte di lavoro, Bufalino presentava la propria esperienza poetica come il tentativo di «far sorridere [...] un fantasma di gioventù ${ }^{7}$, ancora maggiori erano il suo disincanto e distacco alla metà degli anni ' 60 . Sostanzialmente risentito dalla risposta interlocutoria e dalle riserve abbastanza esplicite nei confronti di un'«educazione letteraria» tutt'altro che dissimulata ${ }^{8}$, egli avvertiva una resistenza dei tempi (evidentemente non più quelli della poesia neorealista, ma delle sperimentazioni avanguardistiche) all'anelito mallarmeano di sconfiggere le hasard, conciliando «calcolo e avventura, reticenza e passione». Sembra dunque che l'utopia del libro sia svanita agli occhi di Bufalino, la cui lirica è ora supportata dall'impulso a preservare frammenti di memorie giovanili, ricordi rassicuranti di una permanenza nell'accogliente grembo isolano, da consumato reduce di un apprendistato di morte maturato tra le inquietudini giovanili «all'ombra della guerra».

\section{Gli 'incunaboli' dell'Amaro miele}

5 Ma per ricostruire gli antefatti della poesia bufaliniana, più che guardare ai componimenti apparsi su «Uomo» e «Democrazia», occorre indagare, partendo dalla seconda edizione dell'Amaro miele (1989), sui versi di Rimanenze, sezione che, se per un verso obbedisce a un proposito di documentazione dell'attività versificatoria $\mathrm{e}$ traduttiva dell'autore e contiene una serie di testi esclusi dalla triplice articolazione della princeps, per l'altro conserva le poesie più antiche, riunite in un quaderno che egli chiama «d'anteguerra» (seguito da quelli di guerra e del dopoguerra) ${ }^{9}$. Di questi testi, alcuni dei quali anteriori agli anni ' 40 , è conservata una testimonianza seppur limitata che offre qualche lume sull'officina poetica del comisano.

Rimanenze è in effetti l'unica sezione dell'Amaro miele di cui si conserva una pur minima documentazione redazionale. Si tratta di 20 carte dattiloscritte ${ }^{10}$, prevalentemente utilizzate solo sul recto, con correzioni e varianti manoscritte in inchiostro rosso, blu e nero. Il procedimento di revisione testuale è quello consueto del comisano, tipico anche del dattiloscritto preparatorio della prima edizione ${ }^{11}$, consistente nell'incollare il testo sostituente con scotch, anche se talvolta su questi cartigli sovrapposti egli effettua ulteriori interventi a penna.

7 Nella nota alla seconda edizione l'autore ascrive agli anni 1939-1941 le poesie da Prove a Vedetta notturna. Per il primo di questi componimenti i materiali dattiloscritti rivelano un'incertezza sul titolo: Scarabocchi e frantumi, poi divenuto Detriti e frantumi, e infine Prove (1941) d'anteguerra, che poi darà vita al titolo della sezione e a quello della poesia d'apertura: Quaderno d'anteguerra (1939-1941) Prove. Per la seconda poesia sono 
documentate queste variazioni: Frantumi di poesie perdute (poi disperse), Detriti di poesie non compiute, Mosaico, Pot-pourri.

Da questi dati iniziali, si ha l'impressione che il 'primo tempo' della poesia bufaliniana si sviluppi all'insegna della frammentarietà, di una certa oscillazione tematica che richiede ancora una messa a punto del linguaggio, anche se le titolazioni dei primi componimenti, più che a un ricordo sbarbariano, rimandano a un'idea di incompiutezza, provvisorietà e precarietà della vita e della parola che la dice. Al centro è la donna, vista come figura mitica, datrice di incanti e sortilegi ma al contempo icona persefonea, «ecate d'oro, immagine lucifera» (così definita in In margine a una dispensa di greco).

Mentre i testi del Quaderno d'anteguerra sono anteriori alle tre sezioni dell'Amaro miele 1982, quelli della sezione successiva, da Vedetta notturna a Epigrafi, datati 1942-1945 (la didascalia della prima poesia è «Servizio Unppa, 1942»), attestano una fase in cui la preistoria si contamina con la storia della poesia 'matura'. Qui i dati del vissuto, ricostruibili da luoghi e nomi presenti nei testi (ad es. Lapide per Pietro Carabilló) ${ }^{12}$, lasciano spazio all'autointrospezione, a un colloquio, che non ha più nulla di crepuscolare, con il proprio cuore, alter ego di un vissuto di vagabondaggio e di una condizione 'fuori di chiave': «O mio cuore, episodio / inutile [...] / Come un ospite che nessuno sopporta / vado fra gli uomini, stanco / di chiedere in elemosina la morte» ${ }^{13}$. E sempre il cuore è il destinatario del secondo dei Due epitaffi, che da un iniziale Per me stesso diventa leopardianamente Per se stesso: «Pugno di niente, pacifico cuore, / quanto silenzio infine dentro di te! $»^{14}$.

10 Seguendo quanto l'autore dice nella Nota alla seconda edizione, l'ultimo gruppo di poesie di Rimanenze abbraccia gli anni 1946-1954. C'è in alcuni di essi quell'attesa e persuasione di morte che ispira accenti di congedo dalla vita e dal cuore: «Mia vita, mio cuore, passate: / non voglio udirvi battere più» ${ }^{15}$. La cronologia di questi testi si intreccia con quelli apparsi su «Uomo» e «Democrazia», che diverranno parte della princeps. Nella prima testata compaiono componimenti riversati nella prima sezione: Autunno a Gaiato (confluita con varianti nella terza parte di Fogli dal diario d'inverno), Due anniversari (la prima parte è inedita, la seconda sarà con molte varianti il quarto dei Congedi), Parole di Saulo, Vento di roccia, Ed il tuo arco, i primi due ripresi con lo stesso titolo, l'ultimo espunto ${ }^{16}$. Sul secondo periodico, nel numero dell'11 gennaio 1948, sono stampati Paese (poi in Asta deserta), Verrà l'angelo ladro, O statura delusa, Borea alza la nube di folgore (tutti in Annali del malanno; il secondo diverrà il primo dei Congedi, l'ultimo prenderà il titolo di Malanotte).

11 La storia di questi testi, almeno di quelli consegnati alle riviste, va letta e compresa nella cornice culturale e umana del rapporto epistolare con Angelo Romanò, al quale Bufalino confessa a metà degli anni ' 50 di conservare un «vecchio quaderno» di poesie scritte tra il' 44 e il '50. Sono composizioni che non lo entusiasmano («bellissime, ma solo un giorno l'anno»), che però, liberate «di tanti stenti e goffaggini e corrivi estetismi», potrebbero rivelare «un nucleo di motivi gracili e dolenti» ${ }^{17}$. L'abbrivo di questa scrittura affonda (e non può essere diversamente) in quel côté simbolista proprio della generazione coeva di letterati o aspiranti tali, anche se nelle parole di Romanò si intravedono i rischi e i limiti di un'immersione esclusiva nel «declivio dolcissimo dei maledetti $\aleph^{18}$. Come ha ben evidenziato Nunzio Zago, in Bufalino, che ha già messo a fuoco una visione negativa e disincantata del reale, «quella letteratura rappresenta un punto malfermo ma irrinunciabile di riferimento ${ }^{19}$. Una visione che va di conserto con 
la sofferta e tormentata elaborazione di un pensiero religioso, di sapore gnostico, diversa dall'inquieto esistenzialismo cristiano che Romanò respirava nell'ambiente - fortemente suggestionato da una personalità come quella di David Maria Turoldo di una rivista come «Uomo». Bufalino si presentava con la sua «miscredenza, ferma e candida come una fede», con l'aut aut kierkegaardiano di chi si trova involontariamente costretto a scegliere tra il Bene e il Male, impossibilitato a concepire un Dio «infinitamente giusto e insieme infinitamente buono», e per di più con l'idea della creazione come 'inciampo', frutto di un errore «vergognosamente superfluo» ${ }^{20}$.

Cosciente dell'altissima tensione morale di quel foglio, Bufalino si presentava quale poeta esordiente, con lo scetticismo di chi sapeva di offrire «divertimenti o esercizi che s'arrendono presto alla resistenza della materia ${ }^{21}$. Tuttavia, nei versi inviati dal sanatorio della Rocca nel giugno del ' 46 egli sembra aver elaborato la visione agonistica di una fede senza estetismi di sorta, una cristologia che esprime una sofferta dialettica interiore (ma che sarà poi, all'altezza del primo romanzo, un'imitatio Christi realizzata sub specie aegritudinis, e diverrà al contempo stigma e stemma) ${ }^{22}$. Un differimento della grazia «dopo il diluvio», una convivenza necessaria con questo «scuro e dolce mostro che è la vita». Tutto ciò nell'attesa di una palingenesi, con l'animo del «mendicante caparbio» e del "paralitico» rivisitato con le parole delle rimbaudiane Proses évangéliques. Ai versi è affidata questa disperata speranza di un'attesa, consumata da chi, rispetto all'approdo rassicurante e consolatorio dell'amico, vive la propria vocazione «di triste eresiarca» e intrattiene con la persona di Cristo «un duello funesto e sacro» al tempo stesso ${ }^{23}$.

Poiché questi componimenti saranno sottoposti da Bufalino a una serie consistente e significativa di varianti, riportiamo, per i primi due, la versione rielaborata nella prima edizione dell'Amaro miele:

\begin{tabular}{|c|c|}
\hline Parole di Saulo («Uomo») & Parole di Saulo (AM) \\
\hline $\begin{array}{l}\text { La vipera illesa si torce } \\
\text { sul solco della folgore, che tempi } \\
\text { di laboriosi prodigi: } \\
\text { più tardi un gesto abolirà le rupi. } \\
\text { Ma nell'aria che non dà suono } \\
\text { se bassa sbanda la clava di luce, } \\
\text { che grido mi squarcia la bocca sepolta, } \\
\text { che piaga balza sulle mie palme felici? } \\
\text { Rose aspre sfavillano da siepi, } \\
\text { io curvo sotto il pugno arido tremo } \\
\text { che mi devasta: cuore, } \\
\text { cuore di sangue che dentro mi bruci, } \\
\text { scoppia, è l'ora demente, è l'ora sola. } \\
\text { Già la caccia s'avventa nel silenzio. } \\
\text { Crolleremo con occhi di pietra. } \\
\text { La Sua fionda non sbaglia. }\end{array}$ & $\begin{array}{l}\text { L'illesa angue si torce } \\
\text { dove strisciò la folgore, che tempi } \\
\text { di laboriosi prodigi: } \\
\text { più tardi un gesto abolirà la rupe. } \\
\text { Ma nell'aria di zoccoli e torce, } \\
\text { se sbanda la clava di luce, } \\
\text { se la tromba ripete l'allalì... } \\
\text { scoppiami, cuore, che dentro mi bruci; } \\
\text { balzate, piaghe, sulle mie palme felici! } \\
\text { Questo luogo mi piace per morire. } \\
\text { Cadrò con occhi di pietra. } \\
\text { La Sua fionda non sbaglia. }\end{array}$ \\
\hline
\end{tabular}




\begin{tabular}{|c|c|}
\hline Vento di roccia («Uomo») & Vento di roccia (AM) \\
\hline $\begin{array}{l}\text { Di cieche voci come un'acqua che batte } \\
\text { contro la chiusa e non ha respiro } \\
\text { sempre consolerò le mie deserte } \\
\text { Estati, ora che a valle } \\
\text { in un franare celeste di fiori } \\
\text { strenuo il solstizio si dissenna: non ho } \\
\text { che larve o funeste saggezze, } \\
\text { e questa terra rapinata e dolce } \\
\text { dove gli argini crollano al preciso } \\
\text { contagio della luce, ma nemica } \\
\text { è l'ora che dalle palme s'invola. }\end{array}$ & $\begin{array}{l}\text { Di avare voci, come un'acqua che batte } \\
\text { contro la chiusa e non ha respiro } \\
\text { sempre consolerò le mie giornate } \\
\text { deserte, ora che a valle } \\
\text { fra criniere di fiori di luce } \\
\text { si dissenna il difficile Cane, } \\
\text { e al suo contagio piegano gli enormi } \\
\text { mandriani le nuche di terra, } \\
\text { e il mio trofeo d'esecrata saggezza } \\
\text { si fa nebbia per sempre. }\end{array}$ \\
\hline $\begin{array}{l}\text { Qui s'arresta il mio giro, non ha volto } \\
\text { altra vicenda che di sangue, infine } \\
\text { vento di roccia rompe } \\
\text { la notte e fa tumulto alla memoria. }\end{array}$ & $\begin{array}{l}\text { Qui s'arresta il mio corso, più non voglio } \\
\text { che patire il mio sangue, la mia fine, } \\
\text { bagnare il viso a questo } \\
\text { alto vento di roccia } \\
\text { che turba il cielo e rompe la memoria. }\end{array}$ \\
\hline
\end{tabular}

Rimandando a uno studio separato un'indagine complessiva sulla variantistica della lirica bufaliniana, notiamo solo alcuni elementi inerenti alla dimensione della religiosità che si affaccia da questi testi di notevole difficoltà interpretativa. La parabola esistenziale è vicenda di sofferenza dello spirito e del corpo, e quindi di sangue, che connota soprattutto la prima versione: il «cuore di sangue che dentro mi bruci» diventa "cuore, che dentro mi bruci», mentre la vicenda «di sangue» si muta in un più patetico «non voglio / che patire il mio sangue, la mia fine».

Sono testi che, mentre illuminano il complesso e contraddittorio sottofondo religioso della voce poetica bufaliniana, individuano la necessità esistenziale e letteraria dell'autore: quella che, con una parola meno inquieta e più sorvegliata, Romanò definiva come bisogno di uscire dalla «consuetudine d'antinomie, carne ed anima, inferno e cielo, realtà e sogno»; un liberarsi insomma dai dualismi per giungere a una scelta e porre fine alla pericolosa soggezione, a una kafkiana "aria del carcere», trovando, con montaliano proposito, «la maglia rotta nella rete / che ci stringe» ${ }^{24}$.

\section{Verso il 'primo' libro}

3.1. In una delle prime lettere all'amico commilitone Bufalino, se da un canto svela $i$ suoi precoci abbandoni ai parnassiani e al D'Annunzio pre-alcionio, dall'altro chiarisce con autobiografico sussiego i motivi della sua ispirazione poetica, che verranno a comporre un libro fatto di 'quadri' stagionali come L'amaro miele:

Uscivo dall'adolescenza come da un reame di febbri e d'oro. La vecchia storia: la donna esigua e dolente, strana e dolce, che bisognava toccare. Poi l'ombra squilla e deride. Fu una storia di paesaggi e di stagioni, inoltre. I miei Autunni, delirare immobile del vespro sul povero oro degli orti, il cielo che si sgretola nel vento; le mie Estati, vipere in un grappolo di luce, lapidazione del sole. Infine "le bateau ivre" agonizzato nel rigagnolo ${ }^{25}$. 
Sono appunti anteriori alle lettere mandate dalla Rocca, motivo per cui nel repertorio poetabile (la donna, l'amore, le stagioni, un «battello ebbro» rivisitato) non rientra ancora il tema della malattia. Questo fatto ci riporta alla domanda iniziale: quanto aveva finora scritto Bufalino? Anche in presenza dei testi su rivista, e detratti gli esperimenti poetici giovanili, destinati alla plaquette non venale I languori e le furie ${ }^{26}$, non siamo in grado di stabilire la consistenza complessiva della produzione in versi fino al '48, anzi fino alla metà degli anni ' 50 , quando l'autore confidava a Romanò di avere un manipolo di versi che sarebbero forse potuti divenire un primo libro.

Certo è che, circa un decennio dopo, al momento di inviare ad Anceschi una silloge delle proprie poesie, egli aveva già abbozzato una sistemazione abbastanza organica, $e$ probabilmente, fatta eccezione per le poche liriche scritte dopo, ma di cui non sa indicare la data, non aveva più dato fiato alla propria ispirazione in versi. Quanto arrivava in mano ad Anceschi era comunque una forma seppur embrionale del futuro libro. Di questo ipotetico Ur-Amaro miele si è conservata traccia in un indice dattiloscritto contenuto sul verso di alcune carte di una prima stesura di Argo il cieco $^{27}$, recante sui primi due fogli di guardia l'indicazione autografa Scartafacci, con una serie di appunti tra cui il primo titolo dell'opera, ossia Fiaba nera. Siamo davanti a un vero e proprio palinsesto, in cui Bufalino verga continui rifacimenti, e sulla cui utilità manifesta in limine dei dubbi («Forse qualche frammento serve ancora»). Ma ciò che interessa è che per molti di questi rifacimenti del secondo romanzo egli si serve del verso bianco di fogli contenenti alcune poesie. In parecchi di questi fogli si ripetono, seppur in modo frammentario e solo una volta in maniera integrale, tre poesie (Malincuore, il giorno del santo, "Intermittenze" in via Rosolino Pilo, Il risorgimento) e l'indice stesso, ricostruibile in modo completo attraverso la collazione dei fogli reiterati.

Questo proto-indice risulta ripartito in quattro sezioni, che con spostamenti e accorpamenti delle poesie verranno a formare quelle della princeps. Il numero dei componimenti è inferiore a quello della prima edizione, segno che alcuni testi erano stati scritti in anni successivi, ma senza incidere sostanzialmente su una forma-libro con cui l'autore si presentava già in quegli anni ad Anceschi.

Trascrivo di seguito l'indice, a fianco del quale indico le corrispondenze con i titoli definitivi. Per alcune poesie non è stato possibile rinvenire l'equivalente a stampa (e pertanto si utilizza il punto interrogativo); per altre la ricerca è stata facilitata dal fatto che il titolo diviene talvolta il verso di uno dei testi pubblicati, oppure viene parzialmente ripreso. Col grassetto sono state evidenziate le poesie che faranno parte della 'forma'-prosimetro dell'Amaro miele:

\begin{tabular}{|l|l|l|}
\hline Numero di pag. & \multicolumn{1}{|c|}{ Titolo nell'indice } & \multicolumn{1}{|c|}{ Titolo in Amaro miele 1982 } \\
\hline & \multicolumn{1}{|c|}{ Sezione Prosopopea di Gessica } & \\
\hline 1 & $\begin{array}{l}\text { Esperide } \\
\text { Progetto di lode } \\
\text { Notizie a un amico immaginario }\end{array}$ & $\begin{array}{l}\text { Esperide } \\
\text { Progetto di lode } \\
\text { Confidenza a un amico immaginario }\end{array}$ \\
\hline
\end{tabular}




\begin{tabular}{|c|c|c|}
\hline 2 & $\begin{array}{l}\text { Scioglilingua per piacerle } \\
\text { Frase } \\
\text { Brindisi al faro }\end{array}$ & $\begin{array}{l}\text { Scioglilingua per piacerle } \\
\text { Frase } \\
\text { Brindisi al faro }\end{array}$ \\
\hline 3 & $\begin{array}{l}\text { Patto prima dell'ira } \\
\text { Paese } \\
\text { Imbarcazione serale } \\
\text { Domenica d'un fauno }\end{array}$ & $\begin{array}{l}\text { Patto prima dell'ira } \\
\text { Paese } \\
\text { Barcarola? } \\
\text { Pomeriggio d'un fauno minore (Rimanenze) }\end{array}$ \\
\hline 4 & $\begin{array}{l}\text { Stampa d'Epinal } \\
\text { Ritratto dell'amata come Persefone } \\
\text { Di un difficile oracolo }\end{array}$ & $\begin{array}{l}? \\
\text { In margine a una dispensa di greco } \\
\text { (Prosopopea dell'amata come Persefone) } \\
\text { (Rimanenze) } \\
\text { Di un difficile oracolo }\end{array}$ \\
\hline 5 & $\begin{array}{l}\text { Exergo per una musica barocca } \\
\text { A lei tornata dal sonno }\end{array}$ & $?$ \\
\hline 6 & $\begin{array}{l}\text { Bestiario } \\
\text { Citazione } \\
\text { Emblema } \\
\text { Chalet di Cava d'Aliga }\end{array}$ & $\begin{array}{l}\text { Bestiario } \\
\text { Citazione } \\
\text { Emblema } \\
\text { Ballo a Cava d'Aliga }\end{array}$ \\
\hline & Sezione Quaderno dei congedi & \\
\hline 8 & $\begin{array}{l}\text { Versi lasciati sopra il cuscino } \\
\text { Aubade } \\
\text { Pioggia venuta dal mare }\end{array}$ & $\begin{array}{l}\text { Versi lasciati sopra il cuscino } \\
\text { Aubade } \\
\text { Pioggia venuta dal mare }\end{array}$ \\
\hline 9 & Appuntamento presso un bunker abbandonato & Appuntamento presso un bunker abbandonato \\
\hline 10 & $\begin{array}{l}\text { Saldo alla pensione "Beausejour" } \\
\text { Un segno con l'unghia } \\
\text { Attraversando in treno l'Appennino }\end{array}$ & $\begin{array}{l}\text { Saldo alla pensione "Beausejour" } \\
\text { Un segno con l'unghia } \\
?\end{array}$ \\
\hline 11 & $\begin{array}{l}\text { Cartolina a nessuno } \\
\text { Sinopia della morte }\end{array}$ & $\begin{array}{l}\text { Cartolina militare } \\
\text { Sinopia della morte }\end{array}$ \\
\hline 12 & $\begin{array}{l}\text { A Sesta Ronzon, dovunque si trovi } \\
\text { Svolta } \\
\text { Esito }\end{array}$ & $\begin{array}{l}\text { A Sesta Ronzon, dovunque si trovi } \\
\text { Svolta } \\
\text { Esito }\end{array}$ \\
\hline & Sezione Verbali del malanno & \\
\hline 14 & $\begin{array}{l}\text { Monologo dell'untore } \\
\text { Natura morta alla “Rocca" } \\
\text { Canto d'autunno }\end{array}$ & $\begin{array}{l}\text { Pro memoria } \\
\text { Stanza alla "Rocca" } \\
\text { Aegri ephemeris }\end{array}$ \\
\hline
\end{tabular}




\begin{tabular}{|c|c|c|}
\hline 15 & $\begin{array}{l}\text { Preghiera di mezzogiorno } \\
\text { Didascalie per una visita medica }\end{array}$ & $\begin{array}{l}\text { Preghiera di mezzogiorno } \\
\text { Didascalie per una visita medica }\end{array}$ \\
\hline 17 & Dai $[$ sic] Kindertotenlieder Adelmo & Lapide del bambino \\
\hline 18 & Iscrizioni funebri & $?$ \\
\hline 19 & $\begin{array}{l}\text { Alla madre } \\
\text { Per un'inutile medicina } \\
\text { Versi scritti sul muro }\end{array}$ & $\begin{array}{l}? \\
\text { Per un'inutile medicina } \\
\text { Versi scritti sul muro }\end{array}$ \\
\hline 20 & $\begin{array}{l}\text { Verrà l'angelo ladro } \\
\text { Altri versi scritti sul muro } \\
\text { Su un calendario nuovo }\end{array}$ & $\begin{array}{l}\text { Verrà l'angelo ladro } \\
\text { Altri versi scritti sul muro } \\
\text { Su un calendario nuovo }\end{array}$ \\
\hline & Sezione Asta deserta & \\
\hline 22 & $\begin{array}{l}\text { Stazione notturna } \\
\text { Parole da lontano } \\
\text { Al fiume }\end{array}$ & $\begin{array}{l}\text { Stazione notturna } \\
\text { Parole da lontano } \\
\text { Al fiume }\end{array}$ \\
\hline 23 & In occasione di una notizia letta sul giornale & In occasione di una notizia letta sul giornale \\
\hline 24 & $\begin{array}{l}\text { Un'invidia } \\
\text { Ritorno alla collina } \\
\text { Giorno a Capo Soprano }\end{array}$ & $\begin{array}{l}\text { Un'invidia } \\
\text { Ritorno alla collina } \\
\text { Giorno a Capo Soprano }\end{array}$ \\
\hline 25 & $\begin{array}{l}\text { Sine titulo } \\
\text { Per un insetto ucciso } \\
\text { Figura del Paladino }\end{array}$ & $\begin{array}{l}\text { Sine titulo } \\
\text { Per un insetto ucciso } \\
\text { Figura del Paladino }\end{array}$ \\
\hline 26 & $\begin{array}{l}\text { Costa dell'ibla } \\
\text { Approdo del fantasma } \\
\text { Ipotesi del peccato }\end{array}$ & $\begin{array}{l}\text { Costa dell'Ibla } \\
\text { Approdo del fantasma } \\
\text { Nascita del peccato }\end{array}$ \\
\hline 27 & Aneddoti militari & Aneddoti militari \\
\hline 28 & $\begin{array}{l}\text { Il male della luna } \\
\text { Iniziazione } \\
\text { A se stesso } \\
\text { Bagattella all'antica } \\
\text { Lamento del facile cuore }\end{array}$ & $\begin{array}{l}\text { Il male della luna } \\
\text { Iniziazione } \\
\text { A se stesso } \\
\text { Bagattella all'antica } \\
\text { Lamento del facile cuore }\end{array}$ \\
\hline 30 & $\begin{array}{l}\text { Malincuore, il giorno del santo } \\
\text { "Intermittence" in via Rosolino Pilo } \\
\text { Il risorgimento }\end{array}$ & $\begin{array}{l}\text { Malincuore, il giorno del santo } \\
\text { Intermittenza in via Rosolino Pilo } \\
\text { Il risorgimento }\end{array}$ \\
\hline
\end{tabular}


\begin{tabular}{|l|l|l}
\hline 31 & Suasoria & Suasoria
\end{tabular}

21 I testi elencati sono complessivamente 67, di contro ai 95 della princeps. Tale computo non è però attendibile, in quanto nel dattiloscritto appaiono diversi titoli non identificabili. Se nel caso di un eventuale rinvenimento della silloge inviata ad Anceschi $^{28}$ questi titoli non trovassero corrispondenza con quelli dell'edizione a stampa, essi rappresenterebbero degli extravaganti, il che determinerebbe una riduzione del novero dei testi poi riversati nell'Amaro miele.

Come si vede, la struttura della silloge presenta un'articolazione diversa da quella definitiva: infatti quella che sarà la terza sezione, La festa breve, si trova sdoppiata nelle prime due, Prosopopea di Gessica e Quaderno dei congedi; gli Annali del malanno, che aprono il libro dell' 82, si trovano in seconda posizione come Verbali del malanno; infine Asta deserta, che occuperà la seconda posizione, si trova all'ultimo posto, chiusa dalla poesia Suasoria, che Bufalino, in una provocatoria forma di congedo dalla sua attività poetica a seguito delle riserve del suo interlocutore, trascrive nella lettera ad Anceschi.

Sulle ragioni che potrebbero aver indotto lo scrittore comisano a ripensare l'ordinamento del libro ritorneremo in seguito. Quello che importa notare è che, salvo lievi scostamenti e una sola ripresa da quella che sarà la sezione Rimanenze (ma si tratta di una imitazione-riscrittura dell'Après-midi d'un faune), i pezzi distribuiti nelle varie sezioni corrispondono (ad eccezione dei titoli non identificabili) con quelli contenuti nella princeps. Più numerose le poesie di Asta deserta e La festa breve, che coprono quasi tutto l'arco dei testi dell'Amaro miele, meno quelle dei Verbali del malanno, dove manca circa la metà della futura sezione, anche se gran parte delle poesie dell'Ur-Diceria sono già presenti.

24 L'assenza di un titolo associato a questo proto-indice pone l'interrogativo se e quando Bufalino lo abbia concepito, e soprattutto se esso sia stato differente da quello assegnato nell '82. L'intertestualità interna del titolo è evidente, essendo legata a un componimento della sezione La festa breve: «Come ci brucia in quest'ora le labbra / l'amaro miele della giovinezza ${ }^{29}$. Ma proprio questa poesia manca nella silloge Anceschi; un'assenza significativa se si pensa che essa offre quasi una mise en abîme della raccolta, quintessenziando quanto scritto nella Nota alla prima edizione: «[...] fare per un momento sorridere, ove ne abbia ancora le labbra capaci, un fantasma di gioventù». Un fantasma che suggerisce la labilità di un'esperienza di vita fuggevole, che diviene vana non appena sfiorata, petrarchescamente dolce e amara. La gioventù è un «minuto» da assaporare, da «sorseggiare», come si dice in Argo il cieco, la cui clausola riprende il medesimo campo metaforico: «Vita, più il tuo fuoco langue più l'amo. Gocciola di miele, non cadere. Minuto d'oro, non te ne andare» ${ }^{30}$.

La formulazione ossimorica del titolo si ritrova nel penultimo capitolo di Diceria dell'untore. Qui la pioggia di fine estate è simbolicamente purificatrice di una "grande peste», segno di un male che, scorporandosi, trascina con sé ogni residuo di orgoglio, e con esso «forse, la gioventù». Inutile la «seduzione del nulla», inutile l'infelicità: «E l'infelicità, col suo miele amaro, neppure essa mi serviva più» ${ }^{31}$. È lo stato di chi ha trovato nel sanatorio una condizione di vita autentica, e pertanto vive con un senso di colpa la dimissione, in quanto essa schiuderebbe strade «facili, rumorose, comuni», come anche «mezze fedi», «false bandiere» ${ }^{32}$. 

miele - Diceria dell'untore - Argo il cieco, appare anche in altri luoghi dell'opera bufaliniana: nel Fiele ibleo la musica dei versi virgiliani si accorda «col miele di gioventù che ci saziava la bocca» ${ }^{33}$. Nello stesso scritto Bufalino, ricordando il proverbio Cu agghiutti meli nun sputa feli, ne declina il senso sia morale che letterale, facendo degli Iblei, «luogo di fiele», una metafora della Sicilia-mondo:

E vorrà dire, volgendolo al morale, che non si deve pretendere gentilezza da chi ha masticato amaro tutta la vita. Epperò anche il significato letterale funziona: non poter più resistere officine del miele, là dove il cielo è di fumo, e chiazze di calce $\mathrm{e}$ gesso insozzano il verde, e il sentimento comune, dall'alba al tramonto, è la collera. Non più luogo di miele, gl''́blei, ma luogo di fiele ${ }^{34}$.

Evidente l'ascendenza petrarchesca del titolo: «et non so che nelli occhi, che 'n un punto / pò far chiara la notte, oscuro il giorno, / e 'l mèl amaro, et addolcir l'assentio»

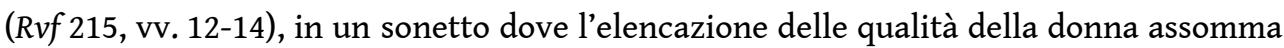
un campionario di virtù tra loro complementari. Tuttavia l'amarezza intrinseca alla condizione amorosa è significata in modo più diretto nella canzone Quell'antiquo mio dolce empio signore, dove si legge: «O poco mèl, molto aloè con fele! / In quanto amaro à la mia vita avezza / con sua falsa dolcezza, / la qual m'atrasse a l'amorosa schiera!» (Rvf 360, vv. 24-27) ${ }^{35}$. Un Petrarca ripreso da Montale (soprattutto quello degli Ossi, libro tanto compulsato da Bufalino nelle sue relazioni epistolari con Romanò, e soprattutto livre de chevet del protagonista di Diceria) nell'osso' breve Mia vita, a te non chiedo lineamenti: «Nel tuo giro inquieto / ormai lo stesso sapore han miele e assenzio». E d'altronde l'acedia petrarchesca serviva a Bufalino per dare consistenza culta alla sua disamina estetica sulla malattia in letteratura: chi ne è afflitto «non è più il malato comune, [...] ma per lo più un intellettuale, in qualche modo un essere d'eccezione, dai nervi sensibili e dalla vorace immaginazione ${ }^{36}$. Ed è in questo 'accarezzare' voluttuosamente, in questo godere dell'eccezionalità e fuggevolezza di questa condizione del malato, in pectore futuro eletto, a fare della letteratura l'amaro miele per antonomasia. Da qui il quadrilatero semantico acedia-amaro miele-giovinezza-letteratura, in cui si inscrive un titolo che è indubbiamente un fil rouge e un perpetuo commento dell'opera omnia di Bufalino.

Che la trafila petrarchesco-montaliana stia alla base del nome del suo libro, come risalita a una tradizione alta e immune dalle spericolatezze avanguardistiche, è dato così naturale da apparire ovvio. Ma la concezione e la grana semantica e tematica Bufalino le mutuava anche da Baudelaire, l'autore con cui ingaggiava un corpo a corpo da traduttore che lo faceva anche acuto lettore delle contraddizioni delle Fleurs du mal, un'opera il cui nodo sta «in uno stigma che cerca dolorosamente di diventare stemma»; dove ogni elemento della natura e la stessa trascendenza di Dio partecipano di una dualità perpetua, cosicché il lettore si trova di fronte a un gigantesco «ossimoro psicologico e morale» ${ }^{37}$. E di questo ossimoro permanente, e della connessa capacità di «estrarre dalla coscienza del Male la "rare fleur" della poesia»" fiele del libro continua a riempirci la bocca» ${ }^{39}$, Bufalino si è senz'altro ricordato, senza rinnegare le illustri ascendenze del 'filo d'oro' della tradizione italiana. Non è certo un dettaglio che nell'Amaro miele vi sia un esplicito omaggio baudelairiano nella poesia Canto d'autunno, originariamente posta quale epigrafe del cap. IV di Diceria, per poi divenire Aegri ephemeris, passando nel dattiloscritto preparatorio per Chant d'automne. Una poesia che, nella sua originaria destinazione, anticipava «il doloroso connubio tra desiderio fisico e morboso corteggiamento dei segni ripugnanti della malattia cui sono 
non a caso costantemente improntati gli incontri trai due tisici protagonisti» ${ }^{40}$ del romanzo.

3.2. Un breve attraversamento tematico delle sezioni di questo 'incunabolo' dell'Amaro miele potrebbe fornirci ulteriori elementi per rispondere alla questione inerente al titolo; un titolo capace, come si è visto, di sviluppare tutto il proprio potenziale semantico quando lo si mette in rapporto con le zone più sensibili dell'opera del comisano.

Le prime due parti, Prosopopea di Gessica e Quaderno dei congedi, confluiranno in La festa breve, la sezione non a caso più corposa della princeps, dove si trovano testi dedicati all'amore e alla donna, con la tipica declinazione bufaliniana di questi temi:

Nella mia opera l'amore è visto generalmente come commedia d'inganni, non nel senso di una frode maligna, ma come cinema di larve, una specie di sogno interrotto e creativo che somiglia al sentimento dell'arte. Con la differenza che non riguarda gli eletti, i vocati ma l'universale [...] Ogni paziente d'amore si comporta, voglio dire, come un romanziere e un poeta e, a somiglianza del primo, edifica personaggi inventati, almeno due, la controparte e se stesso; a somiglianza del secondo, vi tesse attorno metafore e delicate invenzioni. È una guerra emozionante che, come tutte le guerre ha le sue crudeltà e le sue viltà [...]. Per molti versi l'amore coincide con la vita tutta, è spaventoso, stupefacente, incantevole come la vita ${ }^{41}$.

31 In apertura della sezione definitiva risuona, mediato da un titolo leopardiano, il richiamo della vita, del "rumoroso e caro / disordine del mondo» ${ }^{42}$, all'insegna di un mai sopito stupore nei confronti dell'esistenza. Nell'indice dattiloscritto questa poesia si trova nell'ultima sezione, Asta deserta, a prova del fatto che la riarticolazione delle macrostrutture determina anche delle consequenziali migrazioni delle microstrutture.

Il titolo Prosopopea di Gessica, che è anche quello di una delle sezioni dello scartafaccio di Argo il cieco, è fortemente programmatico, a maggior ragione per la presenza in apertura di quella riflessione post eventum sull'amore come tormento e trasgressione che è Esperide. Al posto di questa sezione, che perderà la sua autonomia, si troverà all'interno della definitiva La festa breve una Serenata a Gessica, dove appare una figura di una quotidianità fiabesca («tu da una fiaba mi lanci una rosa»), che come la Silvia leopardiana è «già remota», e viene rievocata nella memoria («ma le palme della sera / l'ingenua fronte bendarti / non senti ancora») e nello stesso tempo congedata come «viso perduto» ${ }^{43}$.

33 A Esperide succede Progetto di lode, che nella princeps formerà un dittico con la precedente Achi lo sa (trasparente riecheggiamento della dedica di Diceria), e che riprende da quella tutto il repertorio biblico-evangelico atto a figurare nella donnadomina un metaforico nutrimento di vita (pane, vino, acqua, grano, roccia, ecc.), ma anche un segno (stendardo nel lessico bufaliniano è emblema di stigma-stemma) da adoperare «contro le scure procedure della sorte» e al contempo «veemente complice di morte» ${ }^{44}$.

34 I testi successivi, ossia Notizie a un amico immaginario (poi Confidenza a un amico immaginario), Scioglilingua per piacerle, Frase (la cui clausola, «Questo è dunque l'amore, e mi spaventa $»^{45}$, ricalca quella del catulliano Odi et amo), Brindisi al faro (dove l'alterità è guarigione da un «maniaco dramma», e dunque riparo dall'ipertrofia dilagante dell'io), Patto prima dell'ira, Paese, Imbarcazione serale (poi Barcarola), presentano una certa omogeneità tematica, a differenza di Domenica d'un fauno (probabilmente trasferito in Rimanenze col titolo Pomeriggio d'un fauno minore) e Stampa d'Epinal, di cui non è noto il corrispondente, i quali sembrano avere una natura più occasionale. Tra i componimenti 
più significativi spicca, per la rete di relazioni testuali che intrattiene con le opere in prosa, Ritratto dell'amata come Persefone, che, stando alla datazione suggerita dall'autore per i versi di Rimanenze (la poesia diventerà In margine a una dispensa di greco, e il titolo originario sarà il sottotitolo), appartiene al Quaderno d'anteguerra, ma che si trova sul verso di una carta dello scartafaccio di Argo il cieco. Ci troviamo dinanzi a un traghettamento testuale assai significativo, ma che costituisce un'eccezione rispetto alla matrice lirica della scrittura di Bufalino: in questo caso non è la prosa a generare il verso, ma la poesia che diviene produttrice di metafore e immagini mitiche, che riguardano sia la figura di Cecilia in Argo il cieco sia quella di Marta in Diceria dell'untore.

Riporto il testo secondo la lezione attestata da Rimanenze, segnando a margine le varianti presenti nella versione dattiloscritta del secondo romanzo:

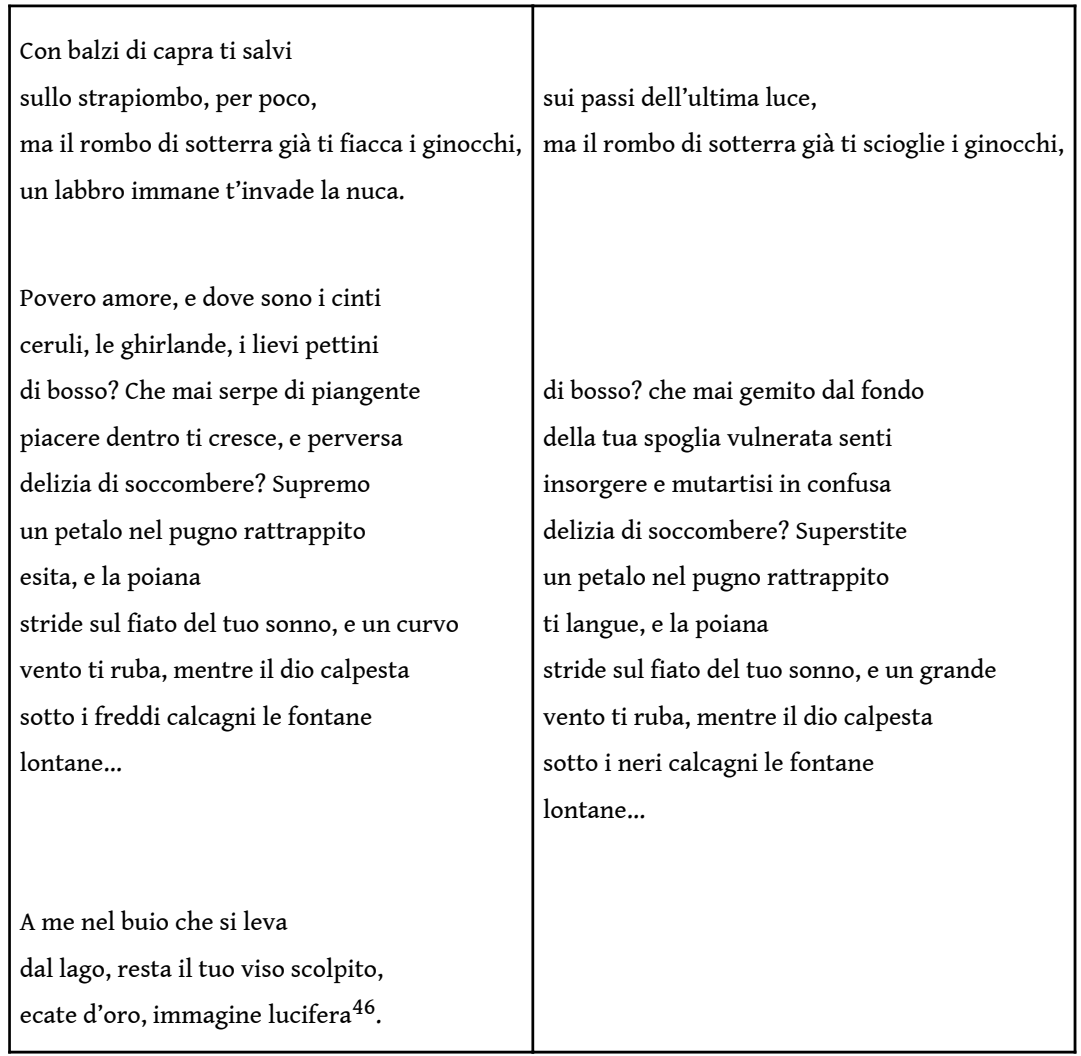

In una delle stesure di Argo il cieco vi è - lo ha segnalato Giulia Cacciatore in una fine indagine genetica - una vera e propria riscrittura prosastica del testo, con riprese pressoché letterali. Nella scena notturna che precede quella dell'amplesso Cecilia dorme e Serafino, guardandola, la paragona alla dea greca Persefone, tracciandone un ritratto con la stessa modalità interrogativa della poesia. Da diverse corrispondenze testuali si vede come la prosa non dipenda dalla stesura dattiloscritta della poesia, ma da quella che sarà stata la versione ne varietur.

Eccola addormentata. Avvolta in una coperta, sul tavolato. Col petto che si alza e si abbassa secondo una metrica scorretta, dove i tempi deboli e i forti s'inseguono in un primo momento precipitosi, ma poi subentra un silenzio così lungo, una specie di morte, che sia morta? Solo che il fiato le torna subito, pacificato, una musica, sembra una tenerezza di vento lungo l'erba di primavera. È viva, dunque, è viva. Invulnerabile nel nascosto circolo del sangue, propria e viva nel suo corpo, dal roccolo di capelli neri appuntato sulla nuca alle unghie dei piedi laccate di rosa. 
Viva, ma dov'è ora? dove la porta il sonno? Somiglia di profilo a quella dea del vaso disseppellito, Persefone o come si chiama. Anche lei come quella, a correre il mondo con balzi di selvaggina, mentre il rombo di sotterra le fiacca i ginocchi, un labbro di dio le invade la nuca. Persefone, e come no? Lei, la povera lodigiana in trasferta, coi suoi quarant'anni ammaccati e bellissimi, il suo profumo d'anima persa. Col medesimo nodo di spaventato piacere che le cresce dentro, la stessa perversa delizia di soccombere. Ma dove sono i cinti, le ghirlande cadute nel sottobosco, i pettini che le tenevano fermi i capelli? Solo uno stelo resiste stretto nel pugno, lo stelo d'un asfodelo. Ma la poiana stride di malaugurio sulle ancelle che scappano, la signorina non torna più. Persefone, dove vai? Che antichi guadi calpesti sotto i freddi calcagni? che corrente ti ruba via? A me, nel buio che sorge umido da questo pozzo di mare solo il tuo volto addormentato risplende, tu fra le braccia e la barba del dio: ècate d'oro, immagine lucifera... ${ }^{47}$

Al Ritratto segue Di un difficile oracolo (presente anche nel citato dattiloscritto di Argo il cieco), che pare segnare un mutamento nella visione dell'amore come unanimità di corpi e destini, in quanto la donna non è più partecipe del patto finzionale, tesa piuttosto a riacquistare la propria (narcisistica) individualità («E mi stupisco ancora / del tuo sangue violento che mi sfida / e sgrida con voce di vento» $\left.{ }^{48}\right)$, fino al distacco adombrato da Chalet a Cava d'Aliga (poi Ballo a Cava d'Aliga):

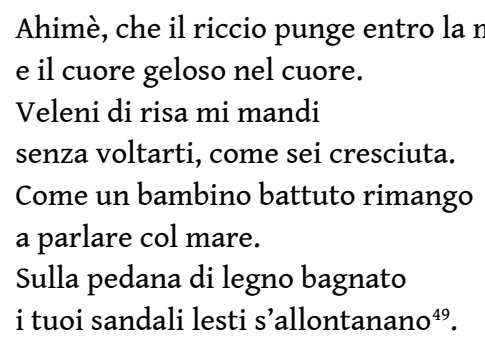

C'è insomma una palinodia della prosopopea, con l'accorta espunzione di testi memoriali, tra cui il'leopardiano' Serenata a Gessica.

La sezione Quaderno di congedi si apre con Versi lasciati sopra il cuscino, che riprende il tema di chiusura di Prosopopea di Gessica, ossia quello della finzione amorosa, ipostasi di quella somma finzione che è la vita. Questa poesia sarà posta da Bufalino a commento del cap. XII di Diceria, dove si narra dell'amplesso tra il protagonista e Marta, a cui seguirà la morte della donna. Le due poesie successive sviluppano questa 'dimissione' dell'amore, questo declinare di un sentimento "che si ammala» ${ }^{50}$, collegato al declino dell'estate, metaforicamente "gioventù menzognera». L'unico regalo possibile è un «regalo di morte», superstite gesto simbolico per chi non riesce più a intessere una relazione autentica, e cerca pertanto una realtà compensativa fatta di presenze fantasmatiche, oppure la solitudine ${ }^{51}$.

Nelle due poesie a seguire (Saldo alla pensione «Beauséjour» e Un segno con l'unghia) a essere in primo piano è l'abdicazione dell'agens-auctor che si appella, con ironia anticrepuscolare, al cuore, invitandolo a chiudere «il libro del suo giorno $»^{52}$. Cartolina a nessuno - forse Cartolina militare? - è collegata a Sinopia della morte dal tema del corpoombra, di un contatto che può sussistere nella sola dimensione del presente: «Relegato il tuo viso dietro un muro / lontano si fa scuro a poco a poco: / non è mai stato, non sarà mai più $)^{53}$.

Dopo un'altra apparizione della donna, musa da coltivare ormai solo nel ricordo, con Svolta il poeta recita l'ultimo e irrevocabile addio alla giovinezza:

Poiché, mentre l'aria rapisce

nel suo sonno le foglie del sangue,

e così piano mi tenta 
quest'esule sole la fronte,

è bello qui fermarsi per dirti addio,

mia giovinezza, mia giovinezza ${ }^{54}$.

La collocazione di Esito, poesia che risalirà tra le prime nell '82, dopo questo congedo dal «miele» della gioventù non è casuale; in essa infatti la perdita della madre sancisce il definitivo distacco dalla terra nutrice: «Tu, madre, anche dal cuore / mi strappi ogni radice $\|^{55}$.

Le altre due sezioni, Verbali del malanno e Asta deserta, costituiscono in buona parteil palinsesto del progettato prosimetro di Diceria, e presentano i componimenti relativi al romanzo in una successione quasi identica a quella delle corrispondenti sezioni della prima edizione dell'Amaro miele. Ciò significa che a metà degli anni ' 60 Diceria era in fase di elaborazione abbastanza avanzata, visto che tutte le poesie scritte alla Rocca coprono 15 capitoli su 17 (e non va dimenticato che tra i titoli ipotizzati da Bufalino vi è anche Annali del Malanno) ${ }^{56}$.

Monologo dell'untore - primo titolo, attestato anche dal dattiloscritto preparatorio dell' Amaro miele, di Pro memoria, a cui arriva passando per Biglietto d'ingresso - si collega alla chiusura del Quaderno dei congedi, reiterando i temi dell'uscita dalla giovinezza e dell'ingresso in una nuova vita. Natura morta alla «Rocca» suggerisce in modo più esplicito del titolo definitivo (Stanza alla «Rocca») un vero e proprio inventario di morte. Dopo Canto d'autunno, di cui si è detto sopra, si trova Preghiera di mezzogiorno, che pur non rientrando nel novero dei componimenti destinati al romanzo sviluppa quella tematica religiosa già enunciata nel I capitolo. Il personaggio-autore, per resistere al rischio di una "cristiana assunzione di colpa» (che ne costituisce lo stigma-stemma) chiede nella poesia un dolore senza narcisismi, in grado di sconfiggere ogni vanità ${ }^{57}$ :

Datemi un male senza libri,

datemi un pianto senza specchi,

una croce che sopra mi vibri,

fatta solo di vento e di stecchi ${ }^{58}$.

In Didascalie per una visita medica il poeta assume le fattezze del Christus patiens, contemporaneamente narratore e protagonista di una «Via Crucis popolare», al cui destino di sofferenza fa da contrappunto la sirena-miele della giovinezza: «E tu pungimi, ape regina, / sotto l'ascella, o mio otre di miele, / anello di mago Merlino» ${ }^{59}$.

46 A Kindertotenlied Adelmo (poi Adelmo e altre lapidi nel dattiloscritto preparatorio dell' Amaro miele, e infine Lapide del bambino) fanno seguito le Iscrizioni funebri, forse corrispondenti al dittico Agli amici morti, e Alla madre. L'omaggio funerario in forma di verso era del resto familiare allo scrittore, che riecheggiando l'Antologia di Spoon River aveva collocato nelle stesure intermedie di Diceria delle Lapidi, "quasi riassunti lacrimosi di ciascun destino nel suo finale esito di cenere», scritti «non senza qualche malizia» e riecheggiando il barocchismo dello stile funerario invalso nel Meridione ${ }^{60}$.

Le poesie successive della sezione (Versi scritti sul muro, Verrà l'angelo ladro, Altri versi scritti sul muro) fanno parte della corona di testi posti a epigrafe del cap. $\mathrm{v}$ del romanzo (si aggiungeranno dopo Compieta e Sine titulo). Si tratta di una zona centrale della cristologia del primo Bufalino, incentrata sulla figura di Padre Vittorio, che costituisce il controcanto di una "vanitosa imitazione di Cristo ${ }^{6}{ }^{6}$ da cui il personaggio protagonista non ha la forza di liberarsi, facendone quello che nel prosieguo di Diceria definirà, col Brancati di Paolo il caldo, Der Christuscomplex, il «complesso di Cristo». 

versi ce la offre Bufalino in Cur? Cui? Quis? Quomodo? Quid?:

Il tema della Sicilia come patrimonio di memorie, mnemoteca e insieme materno ombelico con l'esistenza. Un rapporto difficile: di rigetto, innanzi tutto, per il grumo levantino e facinoroso, per l'intreccio di frode e forza e sole sleale che si suol chiamare mafia, e che mi sforzo ogni momento di censurare e di espellere del mio pantheon di sillabe e sentimenti [...]. Dall'altro lato sento un legame identificatorio, non solo con questo mio triangolo grecocatalano, al di qua dell'Ippari, di cui ho nel sangue i globuli rurali e artigiani di cent'anni fa; ma con l'isola tutta, nella sua complessa mischianza di razze, gerghi, eredità diverse.

Questo dunque è il mio paradosso: di sentirmi per cultura e lingua mentale totus europeus; e di non potermi o volermi scrollare di dosso la pelle Sicilia ${ }^{62}$.

Con Stazione notturna il discorso si apre ancora una volta all'insegna del «commiato»e della «svolta», per poi confluire nella nostalgia del nodo inscindibile di luce e lutto, e quindi delle feste "pompose e intrepide», delle «dissennate serenate», della madre che grida la morte ${ }^{63}$. El'anamnesi della propria vita, custodita nel «triangolo grecocatalano», è affidata, come per il quadruplice fiume ungarettiano, all'«Ippari vecchio», in cui si fondono, nell'intemporale scoperta delle proprie origini, il bianco quasi metafisico dell'infanzia e una morte vestita dei segni del nomadismo, che però per il fiume termina con la fusione nel mare ${ }^{64}$. Segue In occasione d'una notizia letta sul giornale, destinata a commento del cap. XIV di Diceria, dedicato alla morte di Marta, dove il tema portante è la ferita personale ed esistenziale, rappresentata con un irrinunciabile radicamento antropologico.

Un'invidia e Ritorno sulla collina sono testi del nostos, di un'esistenza di comunione corporea con la propria terra-madre che culmina, in Giorno a Capo Soprano, con un desiderio di morte, cosa "facile» e "perfetta» ${ }^{65}$. In sine titulo e Per un insetto ucciso emerge nuovamente il tema religioso, che nella prima poesia si declina come reiterata allocuzione e un Tu trascendente, al Dio che soprattutto in Diceria è soggetto e oggetto di un dialogo contraddittorio e pieno di accostamenti ed elusioni.

Nelle ultime poesie, che sono anche quelle degli ultimi capitoli del romanzo (ad es. Malincuore, il giorno del santo), il cupio dissolvi riaffiora nello stesso segno petrarchesco: «e più m'attempo più voglio morire ${ }^{66}$. Tuttavia la presenza in penultima posizione, anche se in realtà ultima - considerato l'epilogo meta-poetico di Suasoria - di una lirica di riapertura come Il risorgimento, attesta la circolarità esistenziale e biografica che si concentra nella cifra dello stupore, dello sguardo ingenuo sulla vita. Una lirica che costituisce una vera e propria mise en abîme del romanzo, che nell'intenzione autoriale doveva, attraverso la parabola di quell'alter ego che è il protagonista, recare, dopo il rientro «nella vita di tutti», una «educazione alla catastrofe», ma anche «la ricchezza di un noviziato indimenticabile nel reame delle ombre ${ }^{67}$. Il senso di questo stupore, dall'ascesi mitica delle prosopopee, alle Euridici abbandonate (come quella di Delusione d'Orfeo, posta a epigrafe del cap. vi del romanzo, ma non inclusa nell'Amaro miele), fino alle Persefoni addomesticate, attraversa l'Amaro miele senza soluzione di continuità, e sarà la cifra unificante anche dei successivi allargamenti del libro. Questo stupore e incanto stanno interamente in quel titolo emblematico che è Risarcimento, poesia di cui l'autore non sa indicare la data, ma che considera tra quelle 'recenti':

La vita non sempre fa male, può stracciarti le vele, rubarti il timone, ammazzarti i compagni a uno a uno, giocare ai quattro venti con la tua zattera, 


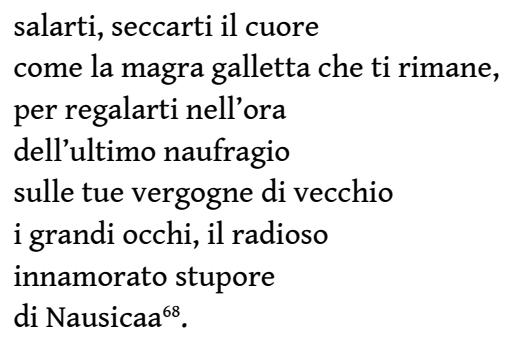

Ci si può chiedere se con l'Amaro miele Bufalino intendesse, in parallelo con Diceria, consegnare il canto di una resistenza della poesia come sistole e diastole del ciclo di illusione (leopardiana) e delusione della vita. Gli interstizi compositivi, le fessurazioni testuali, i 'vuoti' del prosimetro come sintesi dialettica mancata tra prosa e poesia, ne sono probabilmente le attestazioni e silentio più probanti, se è vero che «le parole non servono». È questa la clausola della poesia che chiude la princeps, dove la cifra dell'attesa di un'esistenza che volge al termine («vivo in attesa d'un naufragio») si accompagna all'inchiesta di un Dio pascalianamente "perduto e cercato», «ferito e chiamato ${ }^{69}$, il che inscrive il senso del libro, e il suo stesso titolo ${ }^{70}$, nella tormentosa ricerca di un'alterità in grado di 'risarcire' le ferite dell'esistenza.

\section{NOTE}

1. Istruzioni per l'uso, in G. Bufalino, Opere 1981.1988, introduzione di M. Corti, a cura di M. Corti e

F. Caputo, Milano, Bompiani, 1992, p. 1299.

2. Ivi, p. 1301.

3. Sulla problematica del libro di poesia nel Novecento ci limitiamo a rinviare a N. Scaffai, Il poeta e il suo libro. Retorica e storia del libro di poesia nel Novecento, Firenze, Le Monnier, 2005, con bibliografia pregressa.

4. Nota alla prima edizione, in G. Bufalino, L'amaro miele, Torino, Einaudi, 1996, p. 181. Tutte le citazioni saranno tratte da questa edizione, nella quale, come precisa l'autore, «si aggiunge una raccoltina di Senilia, versi scritti sporadicamente nel corso degli ultimi decenni per occasioni futili e gravi, o anche a supporto di altri testi miei narrativi e aforistici». La sezione Rimanenze, già apparsa nella seconda edizione del 1989, appare "“smagrita" dei non essenziali esercizi di traduzione» (Nota alla terza edizione, ivi, p. 182).

5. Intervista a S.Palumbo, in «Gazzetta del Sud», 18 agosto 1988, cit. in G. Cacciatore, La 'conversione' del '63. Gesualdo Bufalino fra poesia e prosa, in corso di pubblicazione sulla «Rivista di Letteratura italiana», $\mathrm{n}^{\circ}$ 2, 2020, n. 18 (corsivo mio).

6. La lettera è stata scoperta da Giulia Cacciatore, che ne offre la trascrizione integrale e un accurato commento nel saggio citato alla nota precedente. Ringrazio la dott.ssa Cacciatore per avermi messo a disposizione il testo.

7. Nota alla prima edizione, in G. Bufalino, L'amaro miele, cit., p. 181.

8. «Penso anche alla educazione letteraria che Lei mostra. Ma proprio da qui dovrebbe cominciare il discorso. Spero che lo si possa fare; me lo auguro» (lettera del 25 marzo 1963, conservata alla Fondazione Gesualdo Bufalino, segn. 2.12.(1)). La lettera è trascritta nel contributo citato alla nota 5 .

9. F. Caputo, Note ai testi, in G. Bufalino, Opere 1981.1988, cit., p. 1382. 
10. Il titolo di queste carte, conservate presso la Fondazione Gesualdo Bufalino è Le rimanenze. Esercizi di traduzione, segn. MGB. VI (1a).

11. Si tratta di 106 carte dattiloscritte sul solo recto, con correzioni manoscritte in inchiostro nero e rosso, conservate sempre alla Fondazione Gesualdo Bufalino, segn. MGB. VI (1).

12. Su cui v. la lettera di G. Bufalino ad Angelo Romanò del 9 marzo 1945: «m'hanno poi ammazzato un povero amico in nome della legalità» (A. Romanò-G. Bufalino, Carteggio di gioventù (1943-1950), a cura di N. Zago, Valverde (CT), Edizioni Il Girasole, 1994, p. 76).

13. $O$ mio cuore, in G. Bufalino, L'amaro miele, cit., p. 165.

14. Ivi, p. 169.

15. Da tanti anni..., ivi, p. 176.

16. Di seguito l'ordine di pubblicazione: Autunno a Gaiato, $\mathrm{n}^{\circ} 31,6$ aprile 1946; Due anniversari, $\mathrm{n}^{\circ}$

32, 13 aprile 1946; Parole di Saulo, Vento di roccia, Ed il tuo arco, n 38, 1 luglio 1946.

17. A. Romanò-G. Bufalino, Carteggio di gioventù (1943-1950), cit., pp. 200-201.

18. Lettera di A. Romanò del 17 novembre [1943], ivi, p. 24.

19. N. Zago, Introduzione, ivi, p. 14.

20. Lettera di G. Bufalino del 9 marzo 1945, ivi, p. 77.

21. Lettera di G. Bufalino del 16 marzo 1946, ivi, p. 114.

22. Si sofferma su questa lettera, e sulla divergenza di prospettive tra Bufalino e Romanò, il saggio di R. M. Monastra, Bufalino e il linguaggio biblico-cristiano: tra pietà ed empietà, in «Rivista di Studi italiani», vol. XIX, $\mathrm{n}^{\circ}$ 2, 2001, pp. 107-118, alle pp. 110-111.

23. Le citazioni sono tutte tratte dalla lettera di Bufalino del 14 giugno 1946, in A. Romanò e G. Bufalino, Carteggio di gioventù (1943-1950), cit., pp. 129-131.

24. Lettera di A. Romanò del 3 dicembre [1943], ivi, p. 29.

25. Lettera da Sacile del 26-27 novembre [1943], ivi, p. 26.

26. I dattiloscritti preparatori di questa raccolta di juvenilia presentano numerose differenze rispetto al testo poi pubblicato, per via degli interventi di revisione o di vera e propria riscrittura cui Bufalino sottopose i versi nel momento in cui decise di renderli pubblici nel 1995.

27. Si tratta di 341 carte suddivise in due cartelle, segn. ACS1 (cc. 1-134) e ACS2 (cc. 135-341), conservate presso il Centro manoscritti di Pavia. Siamo di fronte a una stesura "più magmatica», costituita da fogli prevalentemente dattiloscritti, con correzioni a penna nera e rossa; altri sono interamente manoscritti, altri ancora contengono rifacimenti realizzati con i consueti cartigli applicati con nastro adesivo (F. Caputo, Note ai testi, in G. Bufalino, Opere 1981.1988, cit., p. 1357). Questa stesura è probabilmente databile alla fine degli anni '70, segno che Bufalino lavorava contemporaneamente ai suoi primi due romanzi (cfr. G. Cacciatore, L'opus perpetuum di Gesualdo Bufalino, in Il miglior fabbro. Bufalino tra tradizione e sperimentazione, Leonforte (EN), Euno Edizioni, 2014, p. 192 e sgg.). È evidente che egli aveva ciclostilato o copiato su carta carbone il fascicoletto dattiloscritto delle poesie e che di alcune di esse aveva fatto uso per lo scartafaccio di Argo il cieco. 28. Di tali riemersioni non è poi tanto remota l'eventualità, essendo tuttora il fondo Anceschi in fase di riordinamento e catalogazione.

29. A media luz, in G. Bufalino, L'amaro miele, cit., p. 83.

30. Argo in cieco, in G. Bufalino, Opere 1981.1988, cit., pp. 269 e 400.

31. Diceria dell'untore, ivi, p. 133.

32. Ibid.

33. Il fiele ibleo, in G. Bufalino, Opere/2 1989.1996, a cura e con introduzione di F. Caputo, Milano, Bompiani, 2007, pp. 1034-1034.

34. Altrove lo stesso proverbio, letto nell'ottica di un moderno contrappasso, diventa immagine della follia che alimenta la creazione letteraria (Altre pagine siciliane, ivi, p. 1159).

35. Le citazioni del Canzoniere sono tratte da F. Petrarca, Rerum vulgarium fragmenta, Edizione critica di G. Savoca, Firenze, Olschki, 2008.

36. G. Bufalino, Da stigma a stemma: il malato come eroe letterario, ivi, p. 1219. 
37. G. Bufalino, Introduzione, in C. Baudelaire, I fiori del male, a cura di G. Bufalino, Milano, Mondadori, 1983, pp. VI-VII.

38. Ivi, p. vi. Il sintagma «rare fleur», dove l'aggettivo è tradotto da Bufalino con «ineffabile», chiude la poesia L'âme du vin (ivi, pp. 198-199).

39. Si cita dall'intervista di M. Onofri, Gesualdo Bufalino: autoritratto con personaggio, in G. Bufalino, Opere/2 1989.1996, cit., p. 1344.

40. M. Paino, Dicerie dell'autore. Temi e forme della scrittura di Bufalino, Firenze, Olschki, 2005, p. 48 (sul rapporto con Baudelaire cfr. le pp. 47-50).

41. G. Bufalino, Cur? Cui? Quis? Quomodo? Quid?, Atti del wordshow-seminario sulle maniere e le ragioni dello scrivere, Taormina, Edizioni di Agorà, 1989, p. 92.

42. Il risorgimento, in G. Bufalino, L'amaro miele, cit., p. 77.

43. Ivi, p. 97.

44. Ivi, p. 91.

45. Ivi, p. 99.

46. G. Bufalino, L'amaro miele, cit., p. 156.

47. Si tratta di una stesura conservata presso il Centro manoscritti di Pavia, siglata AC2, c. 34r. In una delle versioni di Diceria Marta è descritta come un'«esclusa, anima persa» e per questo, nella scena della sua morte, lo scrittore richiama proprio la dea Persefone, citando l'ultimo verso della poesia: “"Marta”, cominciai "Marta, ascoltami” dissi. "Dove sei ora, Marta" chiesi, "dove cammini? In quale notte? Con che nome mi chiami, con che nome devo chiamarti? Ci sono fiumi dove abiti ora? da varcare a nuoto? su passerelle vertiginose? Sei sola? Siete tanti? E di me ti ricordi ancora? Tornami in sogno, Marta. Anche se l'aria duole come basalto sotto i tuoi piedi scalzi. Anche se non trovi labbra per dirmi le parole che vuoi. Guarda come mi lasci in mezzo alla via: una guasta semenza, una sconsacrata sostanza, un pugno di terra su cui casca la pioggia..." [...] Spensi la luce, per non vederlo più, e nella stanza, al chiarore della luna, tornai a cercarla con gli occhi: sembrava dormire, come nella cuna d'una illesa natività; e ancora una volta l'incurvatura dei brevi biondi capelli le componeva attorno all'esangue viso un'aureola da leggenda. // Ecate d'oro, immagine lucifera...» (Centro manoscritti, stesura siglata DUA, cc. 86r, 87r). G. Cacciatore, «Il romanzo multiplo». Étude génétique des œuvres de Gesualdo Bufalino, Thèse dirigée par Christian Del Vento et codirigée par Alberto Cadioli, Université Grenoble Alpes et Università degli Studi di Milano, febbraio 2018, pp. 339-340.

48. G. Bufalino, L'amaro miele, cit., p. 98.

49. Ivi, p. 103.

50. Pioggia venuta dal mare, ivi, p. 105.

51. Appuntamento presso un bunker abbandonato, ivi, p. 111.

52. Un segno con l'unghia, ivi, p. 109 (corsivo mio).

53. Cartolina militare, ivi, p. 113.

54. Ivi, p. 117.

55. Ivi, p. 23.

56. L'elenco di titoli alternativi, che si apre con Annali del malanno e chiude con Diceria dell'untore, è contenuto in un foglio sciolto non numerato accluso all'ultima stesura di Diceria, denominata $G$ dall'editore dell'opera omnia. In questa stessa stesura, ai fogli 148-155 sono riportate, Dal Quaderno'46 intitolato Falsa testimonianza, 11 poesie, alcune delle quali presenti nella prima redazione, contrassegnata con A (cfr. F. Caputo, Note ai testi, ivi, pp. 1330-1331).

57. G. Bufalino, Diceria dell'untore, ivi, p. 11.

58. G. Bufalino, L'amaro miele, cit., p. 9.

59. Ivi, pp. 11-12. La prima citazione è tratta dalla nota d'autore.

60. Lapidi ricopiate, in G. Bufalino, Diceria dell'untore. Nuova edizione accresciuta da pagine inedite e dagli archivi dell'opera. Prefazione di F. Caputo. Con un'intervista di L. Sciascia, Milano, Bompiani, 1992, p. 157. 
61. Istruzioni per l'uso, in G. Bufalino, Opere 1981.1988, cit., p. 1297. Sulla centralità della figura di Padre Vittorio come espressione della religiosità autentica del romanzo sono fondamentali le pagine di A. Sichera, Echi scritturali e lessico religioso nel primo Bufalino, in Il miglior fabbro. Bufalino tra tradizione e sperimentazione, cit., pp. 85-104, alle pp. 99-103.

62. G. Bufalino, Cur? Cui? Quis? Quomodo? Quid?, cit., pp. 74-75.

63. G. Bufalino, L'amaro miele, cit., p. 72.

64. Al fiume, ivi, p. 55.

65. Ivi, p. 66.

66. Ivi, p. 70. Cfr. Rvf 37, vv. 15-16: «Questa speranza [di rivedere Laura] mi sostenne un tempo: / or vien mancando, et troppo in lei m'attempo». Attemparsi è un hapax del Canzoniere.

67. Istruzioni per l'uso, in G. Bufalino, Opere 1981.1988, cit., p. 1342.

68. G. Bufalino, L'amaro miele, cit., p. 180.

69. Poscritto dopo molti anni, ivi, p. 120.

70. Una suggestione fondamentale in questa prospettiva viene dallo studio di A. Sichera, il quale individua una convincente derivazione dall'Introduction à la vie dévote di Francesco di Sales, libro citato nel carteggio tra Bufalino e Romanò, utilizzato più volte nel capitolo di Diceria dedicato a Padre Vittorio, oltre che presente (in una stampa dell'editore parigino Nelson, ma senza data) con alcuni segni a matita e piegature di pagine nella biblioteca dello scrittore comisano. Nel cap. III, intitolato De la patience, si legge: «Ricordatevi che le api quando fanno il miele, vivono e mangiano cibi molto amari, e che allo stesso modo noi non possiamo fare atti di maggiore dolcezza e pazienza, né meglio produrre il miele di un'eccellente virtù, se non mentre mangiamo il pane d'amarezza e viviamo fra le angosce. E come il miele del fiore di timo, erba minuta ed amara, è il migliore di tutti, così la virtù che si esercita nell'amarezza delle più vili, basse e abiette tribolazioni è la più eccellente di tutte» (A. Sichera, Echi scritturali e lessico religioso nel primo Bufalino, cit., pp. 98-99).

\section{RIASSUNTI}

Il contributo, partendo dall'analisi di un proto-indice dell'Amaro miele contenuto in una prima stesura di Argo il cieco, e utilizzando le indicazioni fornite dall'autore nei materiali preparatori di Diceria dell'untore, fornisce delle ipotesi sulla genesi e sulla organizzazione testuale di quello che costituisce non un semplice aggregato di testi, ma un vero e proprio libro di poesia, progettato dall'autore, che solo in un secondo tempo intreccia la propria dinamica interna con la forma 'vitanovistica' del romanzo dell' 81.

Cette contribution prend comme point de départ un brouillon de table des matières d'Amaro miele qu'on a retrouvé parmi les feuillets d'une première ébauche du roman de Bufalino Argo il cieco, ainsi que les indications fournies par l'auteur dans le matériel préparatoire de Diceria dell'untore (1981). Sur la base de ces deux sources d'information on a pu avancer des hypothèses sur la genèse et sur l'organisation textuelle, qui n'est pas un simple assemblage de textes, mais un « livre », conçu en tant que tel par l'auteur. Ce n'est que plus tard que la dynamique interne du «livre» fut temporairement suspendue et déviée par le projet de donner au roman Diceria dell'untore (finalement publié sous une autre forme) une forme de prosimètre, sur le modèle de la Vita nova de Dante, en y incluant les poèmes de L'amaro miele. 
The point of departure of this contribution is a provisional index of L'amaro miele that was found in the first draft of Bufalino's novel Argo il cieco. The essay also relies on an instruction sheet belonging to some preparatory papers relating to Diceria dell'untore (1981). On the basis of this archival evidence, a hypothesis is made on the textual organization and genesis of a work which is not just a gathering of texts but a book of poems, conceived as such by its author. Only later was the internal dynamics of L'amaro miele temporarily modified by the project to use its poems in order to give a prosimetrum form (based on Dante's Vita Nova model) to Diceria dell'untore, a novel that was finally published in 1981 under a different form.

\section{INDICE}

Mots-clés : Gesualdo Bufalino, poésie du XXe siècle, macrotexte, philologie d'auteur Keywords : Gesualdo Bufalino, 20th century poetry, macrotext, author philology

Parole chiave : Gesualdo Bufalino, poesia del Novecento, macrotesto, filologia d'autore

\section{AUTORE}

\section{ANTONIO DI SILVESTRO}

Università di Catania 